\author{
Saroj Kumar Bhattacharyya \\ Patrycja Maciejewska \\ Lars Börger \\ Manfred Stadler \\ Akif Mehmet Gülsün \\ Hasan Basri Cicek \\ Helmut Cölfen
}

\section{Development of a Fast Fiber Based UV-Vis Multiwavelength Detector for an Ultracentrifuge}

Saroj Kumar Bhattacharyya •

Patrycja Maciejewska · Helmut Cölfen ( Department of Colloid Chemistry, MPI Research Campus Golm, Max Planck Institute of Colloids and Interfaces, Am Mühlenberg 2, 14424 Potsdam, Germany

e-mail: Coelfen@mpikg.mpg.de

Lars Börger - Manfred Stadler Polymer Research Laboratory, BASF AG, G201, 67056 Ludwigshafen, Germany

Akif Mehmet Gülsün · Hasan Basri Cicek Department of Electrical and Electronics Engineering, Bilkent University, 06800 Bilkent Ankara, Turkey

\begin{abstract}
The advantages of simultaneously detecting multiple wavelengths in ultracentrifugation experiments are obvious, especially for interacting systems. In addition, the detection of the wavelength dependence of turbidity opens up the possibility to obtain independent information on the particle size in addition to the usual sedimentation coefficient distribution for colloidal systems. We therefore made an effort to develop a fast UV/Vis detector, which is able to simultaneously detect the range from $200-800 \mathrm{~nm}$. This is possible by the use of a modern CCD chip based generation of UV-Vis spectrometers, which translates the dispersed white light onto a CCD chip, where each pixel corresponds to a particular wavelength. In addition to the simultaneous detection of a large number of wavelengths in the range $200-800 \mathrm{~nm}$, also with non integer values, these spectrometers are very fast. Current typical spectrum scan times with the necessary scan quality in the ultracentrifuge are in the range of $100 \mathrm{~ms}$ but this time can be significantly shortened down to $3 \mathrm{~ms}$ for higher light intensities and even down to $10 \mu$ s for a new generation of CCD chip based spectrometers.

The introduction of a fiber based UV-Vis optics into a preparative XL-80K ultracentrifuge with the associated hardware developments
\end{abstract}

will be described as a first generation prototype. In this study, we use a wavelength dependent optical lens system instead of the necessary but more complex wavelength independent mirror optical system for a first check on possibilities and limitations of the optical system. First examples for biopolymers and latexes will be presented and compared to those obtained in the commercial XL-A ultracentrifuge. Already the fast detection enables completely new possibilities like the determination of a particle size distribution in a few minutes. Multiwavelength detection at constant position in dependence of time will be demonstrated, which is an important mode for the use of speed profiles for very polydisperse samples. Also, the use of radial multiwavelength scans will be demonstrated producing a three dimensional data space for monitoring the sedimentation via radial scans with multiwavelength detection. However, despite the advantages, the current problems with the detector will also be discussed including the main problem that much intensity is lost in the important UV range as a result of fiber coupling and bending.

Keywords Analytical

Ultracentrifugation - CCD based UV-Vis Spectrometer - Detector Development · Fiber Optics 


\section{Introduction}

The development of optical detection systems for ultracentrifuges is as old as Analytical Ultracentrifugation itself and dates back to the development of Analytical Ultracentrifugation [1]. The classical detection systems are UVVis absorption optics, Rayleigh Interference optics and Schlieren optics as discussed in detail in the classical book of Lloyd [2]. Furthermore, fluorescence optics was developed for the platform of the Beckman Model E [3] and more recently for the platform of the Beckman XL-I ultracentrifuge $[4,5]$. In addition, turbidity optics was developed for the characterization of particle size distributions of colloidal particles [6-9]. Despite some progress on the detector or illumination part of the optical systems concerning the transformation of photographic detection to CCD chip detection for Schlieren and Interference optics or the use of laser light sources [10-18] (for further references see also the references for multiplexer systems) and photomultiplier detection for the UV-Vis [19] and fluorescence optics $[3,4]$, the classical optical systems remained essentially unchanged for decades. Exceptions are the Flossdorf optics [20], which significantly increased the detected intensity for UV-Vis optical systems and the ultrasensitive Schlieren optics [21].

However, the demands of Analytical Ultracentrifugation have greatly improved with the advent of cheap and powerful computer resources. The most obvious change is the speed of data acquisition and the amount of experimental data becoming available from computer based detectors, which has found its commercial expression in the launch of the Beckman XL-I ultracentrifuge in the 90s of last century [19]. This has in turn catalyzed many methodic advances, which enable analyses with an accuracy not being even imaginable a decade ago [22-26]. However, it must clearly be stated that the development on the data evaluation side is by magnitudes more significant than the hardware development - especially that of optical detection systems for the Analytical Ultracentrifuge (AUC). This is understandable as hardware development is by factors slower than software development due to the delivery and workshop manufacturing waiting times. Nevertheless, there is an urgent need for improved multi detector development for the AUC [27], not only concerning detection speed but also detection capabilities. Examples for the benefit of multi detector use are manifold. They include determination of extinction coefficients [28], even in complicated mixtures [29] but also the possibility of global analysis by evaluation of multiple experiments [30-32] as well as increased experimental information from a single experiment with multiple species and different chromophores by multiwavelength analysis. The detection of multiple wavelengths from UV-Vis absorption optics proved especially useful for the analysis of biopolymers from sedimentation equilibrium [33] or sedimentation velocity [34], although in the reported cases, the analysis was restricted to the three possible scanning wavelengths of the XL-I AUC under addition of information from the interference optical system. Thus, the potential benefit of multiwavelength analyses of hundreds of wavelengths is obvious not only for interaction analyses of complicated interacting polymer mixtures but also for colloidal systems as the wavelength dependence of the turbidity of colloidal samples contains information about their particle size according to the MIE scattering theory [35-38]. With the advent of global analysis approaches involving AUC data, multiwavelength analysis will become an especially important technique for all light absorbing samples of colloidal or polymeric nature.

Parallel to the above developments in AUC methodology, there was also a significant development in the UVVis spectrometer technology. Modern UV-Vis spectrometers can use simple CCD line arrays for the simultaneous detection of a large number of wavelengths, which is essentially only limited by the CCD array pixel number as well as the quality of the applied diffraction grating for the dispersion of the incoming white light. These spectrometers do not only allow for the simultaneous detection of the entire UV-Vis wavelength range, but are furthermore very fast and cheap. Commonly available spectrometers like the USB2000 by Ocean Optics allow for scanning times of down to $3 \mathrm{~ms}$ for an entire UV-Vis spectrum [39]. More recent instruments like the Ocean Optics HR4000 [40] or the series of CCD and ICCD based spectrometers available from LOT-Oriel [41] are magnitudes faster and can scan UV-Vis spectra as fast as $10 \mu \mathrm{s}$. The cooled spectrometers give a quite broad linear dynamic range with the advantage of working with low dark current due to cooling [41]. However, although these fast and powerful UV-Vis detectors are available, they were not yet applied as detectors for AUC's. One of the reasons is that these detectors are based on fiber optics and also operate with incoming white light. White light with wavelength dependent refractive indices makes the precise use of a refractive optics impossible at least in the UV range, where the conventional achromat lenses cannot be applied or vice versa in the visible range if an expensive UV achromat lens system is applied. This is only one obstacle, with no parallel in previous AUC optics, which always operates with monochromatic light - or in case of the XL-A AUC with a torroidally curved diffraction grating producing monochromatic light. This means that in AUC history, the challenge of a multiwavelength optics has not been undertaken despite the potential benefits. This was the starting point for the cooperation between the Max-Planck Institute of Colloids and Interfaces and the polymer physics department of BASF R\&D. This study presents the first results from this cooperation aiming to improve the AUC capabilities by the development of new detection systems. We have on purpose started with the use of conventional wavelength dependent refractive index based lens optics as: 
1. Ocean Optics as spectrometer supplier also offers optimized lens systems. They are available as plug \& play components for a fiber based optical system so that potentially, a fiber optical plug and play system could be applied using the commercial spectrometer software as a first data acquisition software. With this system, first experiences can be made for the further detector development.

2. The mirror optics that use small focal length spherical and parabolic mirrors are not standard optical components, and are not yet available for this application.

3 . Fiber optics are only very rarely applied in AUC detectors. Therefore, potentials and limitations of this optical transport medium for AUC detectors shall be explored.

4. The detector hardware needs to be developed. A lens based system is relatively compact but also the most distorting optical system for white light. Nevertheless, first developments can be made with this compact setup. The adaptation to the mirror based system can be done on the basis of these experiences if necessary.

As a consequence, the present study can only be seen as the first step towards a fast fiber based UV-Vis multiwavelength detector. Here we will only use classical lens optics as a case study well knowing about the problem of chromatic abberation. It is clear that this design cannot be the final prototype but there is already much to be learned not only about the actually detected light intensity but potential problems that may come in way for using other components as fibers or fiber coupled spectrometers. We have therefore also included developments, which are independent of the fiber optical system or multiwavelength detector so that we report about a fiber based high power Xenon lamp as well as on an advanced multiplexer hard/software based on earlier hardware developments [42] currently available as a LABVIEW software. Although just being a snapshot of an ongoing development process, the present study will be able to show the possibilities but also the first limitations of a fast fiber based UV-Vis detector for the AUC.

\section{Experimental}

For the detector development, we used the following commercial products: (a) Spectrometer: USB 2000 Fiber Optics Spectrometer (UV/Vis), Ocean Optics BV, Duiven, The Netherlands with SMA 905 fiber optics connection, $25 \mu \mathrm{m}$ entrance slit, grating: 600 lines $/ \mathrm{mm}$, L2 detector collection lens with UV optimized coating and OFLV-350-1000 2-nd order filter installed and operable with the Ocean optics OOIBASE 32 software. For the UV optimized spectrometer, we have applied a 1200 line holographic grating in combination with a $50 \mu \mathrm{m}$ slit as the entrance aperture (Fig. 5, component 3) and all other UV optimized compounds as described above. (b) Fibers: $600 \mu \mathrm{m} \mathrm{UV} /$ Vis fibers in four channel vacuum feed through, OZ optics, Canada for illumination optics. $600 \mu \mathrm{m} \mathrm{UV} /$ Vis patchcord from Oceanoptics, Duiven, The Netherlands. (c) Focuser: Receptacle style focuser from OZ optics, Canada with biconvex lens $(f=15 \mathrm{~mm})$ and SMA 905 connector (d) Linear actuator: Linear actuator model T-LA28-SV (shorter version) with necessary power supply from Zaber, Canada for precise movement of the detector arm. (e) Lenses and Prism: Biconvex lenses $f=20.6 \mathrm{~mm}$ for custom built collimation optics and $f=20 \mathrm{~mm}$ for collecting transmitted UV/Vis light and a right angled prism from Linos, Göttingen, Germany. (f) Slit: $25 \mu \mathrm{m}$ slit from slit-lens assembly of commercial Beckman XL-I, Analytical Ultracentrifuge. (g) Light source: Xe-Flash lamp with mirror (model L-4633-01) with the relevant power supply from Hamamatsu Photonics $\mathrm{GmbH}$, Herrsching, Germany. (h) Optical Bench: From Owis $\mathrm{GmbH}$, Göttingen, Germany with possibility for $x, y, z$ adjustment and angular adjustment for coupling the light from the flash lamp to fiber. (i) Optical stage: From OWIS GmbH, Staufen, Germany with possibility to fine-adjust in $x$ and $y$ direction for the precise adjustment of the detection optics part of the detector arm. (j) Electrical feedthrough: 18 pin electrical feedthrough for electrical connection of the linear actuator from Lemo Electronic $\mathrm{GmbH}$, Munich, Germany.

Samples for measurement: The proteins were obtained from Sigma-Aldrich, Germany, the latexes were prepared in house. For data generation with the designed detector the following samples were used: (1) BSA $(\sim 1.0 \mathrm{~g} / \mathrm{L})$ in $0.1 \mathrm{MNaCl}, \mathrm{pH}=7.5$. (2) Cytochrome $\mathrm{C}(\sim 1.0 \mathrm{~g} / \mathrm{L})$ in $20 \mathrm{mM}$ MOPS pH 7.2 containing 2 microM $\mathrm{K}_{3} \mathrm{Fe}(\mathrm{CN})_{6}$ and $5 \mathrm{mg} / \mathrm{mL}$ dodecyl maltoside. (3) Polystyrene Latex with different particle sizes $(150 \mathrm{~nm}, 190 \mathrm{~nm}, 240 \mathrm{~nm}$, $430 \mathrm{~nm}, 450 \mathrm{~nm} \& 660 \mathrm{~nm}$ ) were mixed and prepared by dilution with water so that satisfactory intensity of the UV/Vis transmitted light can be detected. (4) For the experiment corresponding to Fig. 12, Polystyrene latex particles of sizes $190 \mathrm{~nm}, 305 \mathrm{~nm}$ and $605 \mathrm{~nm}$ were mixed in a ratio of $1: 2: 2$ by wt. and diluted with water to suitable dilution for the AUC UV/Vis absorption optics.

\section{Results and Discussion}

\section{Hardware Developments}

Illumination System. The whole optical system was designed as a modular system, which permits the use of as many commercially available components as possible and simultaneously enables easy exchange of individual components. For illumination, a Hamamatsu L4633-01 flash lamp was used with a built in reflector which focuses the white light directly into an optical fiber. For further details, see [43]. This design enables a fourfold greater relative light intensity relative to the conventional Hamamatsu 


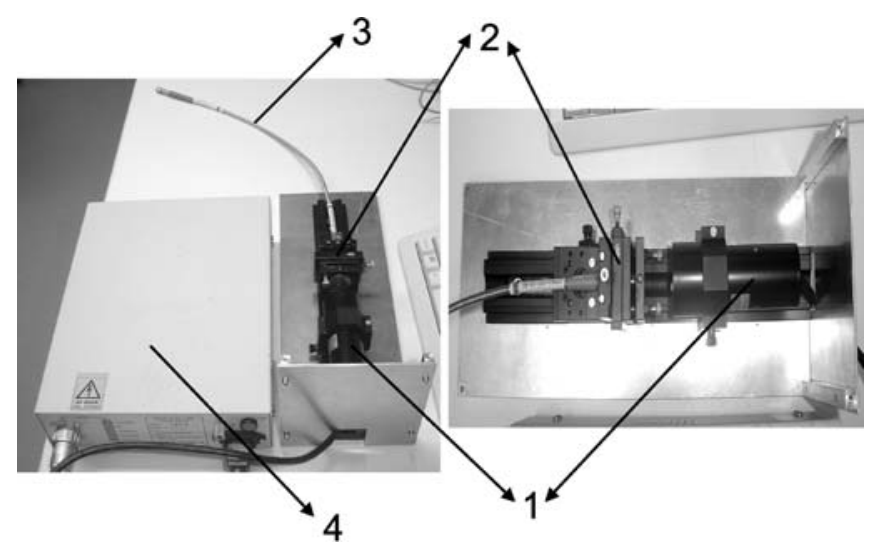

Fig. 1 Set-up of the illumination system for the fiber optics. (1) Lamp, (2) Holder with $x, y, z$ positioning unit, (3) Fiber, (4) Power supply

L-2435 flash lamp, which is installed in the XL-I. However, the main intensity gain is obtained from the fact, that much of the light is coupled into an optical fiber, whereas in the design of the Beckman XL-A AUC, a pinhole in front of the flash lamp is applied as optical aperture, so that much of the light does not enter the optical detection system. A direct intensity comparison between the fiber coupled Xenon flash lamp and the XL-A design in the wavelength range $400-800 \mathrm{~nm}$ revealed a factor of 35-70 higher intensity at the end of the fiber set-up depending on the applied wavelength. However, the flash repetition speed of the flash lamp is relatively slow (only $100 \mathrm{~Hz}$ ), which corresponds to a maximum flash repetition of $10 \mathrm{~ms}$. Although this is the same speed as the lamp of the XL-A, it is not satisfactory in view of the fast spectrometer detection capabilities. If it is considered that a single flash is only about $4 \mu \mathrm{s}$ long, but the flash repetition takes $10 \mathrm{~ms}$, it is clear that the flash lamp is the time limiting component in the present set-up at rotor speed $>6000 \mathrm{rpm}$.

The set-up of the illumination system for the fiber optics is simple. An optical bench (OWIS GmbH, Staufen, Germany) with an $x, y, z$ positioning element is used to mount the lamp and the fiber as shown in Fig. 1.

This set-up enables the optimum coupling of the light from the lamp into a fiber, which can be adjusted by observing the beam intensity exiting the fiber on a sheet of paper. Coupling is then further optimized using the intensity reading from the spectrometer.

Vacuum Feedthroughs/Heatsink Modification. One of the most significant changes of the AUC hardware necessary for the fiber based UV-Vis optical system is the need to feed light and electricity into and out of the vacuum chamber. In the present set-up, this is solved by drilling 6 holes into the heatsink of the XL ultracentrifuge, which was done in the mechanical workshop of the BASF (see

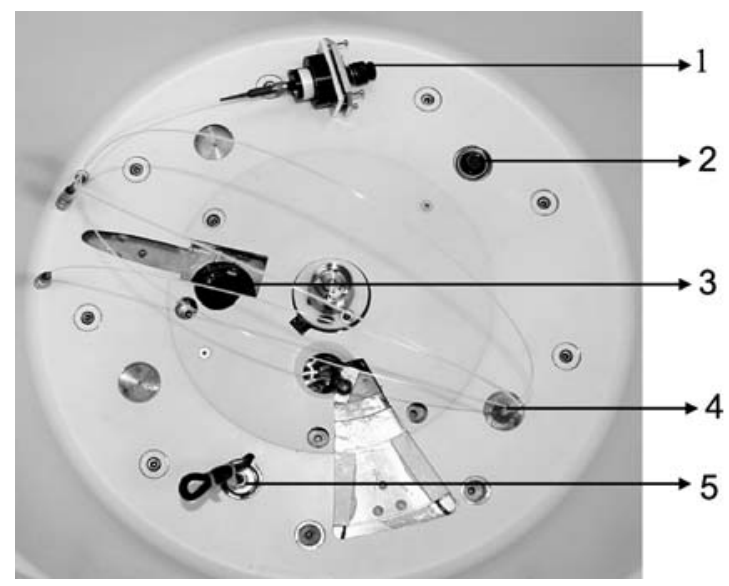

Fig. 2 XL-heatsink with vacuum feedthroughs and mounted fibers. (1) Focuser (2) Vacuum feedthrough for electrical cables (3) Window for further optical system (4) vacuum feedthroughs for fibers (5) Vacuum feedthrough for electrical cables

Fig. 2). This has the significant disadvantage that the Beckman warranty is lost so that future designs will be based upon XL-I heatsinks. However, modified heatsinks of the shown type are operated already for several years in the AUC laboratory at BASF AG, Ludwigshafen, Germany without any problem so that they are considered by the authors to be safe. In addition, the present design in principle allows for the mounting of three detector arms of the type presented in this work so that up to three detectors can be run simultaneously. As vacuum feedthroughs, either a four fiber $600 \mu \mathrm{m}$ vacuum feedthrough from $\mathrm{Oz}$ Optics, Ottawa, Canada was applied as shown in Fig. 2 or one fiber vacuum feedthroughs with SMA 905 connectors (Ocean optics B.V., Duiven, The Netherlands). Both feedthroughs have their specific advantages and disadvantages. Whereas the one fiber feedthrough with SMA 905 connector is a very easy and modular system, it looses light $(\sim 5 \%)$ as it creates an additional two fiber couplings.

The four fiber feedthrough does not have this disadvantage. It also uses only one feedthrough for four fibers and should be the system of choice. However, if a fiber breaks, the whole feedthrough has to be taken out and be sent for repair, which can be a significant disadvantage at least in the prototype design process, where many modifications of the optical system are carried out.

The feedthroughs for electrical cables are less problematic. Here, we use LEMO Electronic, Munich, Germany electric feedthroughs for 18 cables, where one feedthrough is sufficient for the whole UV-Vis fiber optics.

Optical Fibers. The choice of optical fibers is difficult and always a compromise. On one hand, they should enable the transmittance of as much light as possible, while on the other hand, the precision of the optical system is determined by the fiber diameter, as this is the smallest spot size, the light can be focused to. Another requirement is 
that the fibers should have a high transmittance over the entire UV-Vis range (200-800 nm). We have decided to allow for the maximum light intensity, meaning that we use $600 \mu \mathrm{m}$ or even $1000 \mu \mathrm{m}$ fibers (Oz optics or Ocean optics). Another issue, which should not be neglected is the capability of the fibers to bend without too much light intensity loss, especially in the UV range, as the fibers have to be bent in the vacuum chamber in the present prototype. As connector system for the fibers, we have applied SMA 905 connectors, which appear to be the most universal for our purpose, but other connectors are also possible.

The Detector Arm. The detector arm is the central element of the UV-Vis optical system, as it contains all essential optical components of the optical system itself. The detector arm is schematically shown in Fig. 3.

The fiber coming from the lamp is coupled to a collimation system (2 in Fig. 3). This collimation system focuses the light onto the center of the cell. However, it has to be mentioned that the focusing is only achieved for the visible part of the white light and that unavoidable errors are made as long as lenses are used due to chromatic aberration. This can partially be compensated for by using achromatic lenses but they are only available for the visible or UV range respectively and not over the entire range between $200-800 \mathrm{~nm}$, which is envisaged in our application. Therefore, we did not use achromats for this prototype. Instead, we have focused the light onto the center of the cell (spot size ca. $2 \mathrm{~mm}$ ) for the reason that we were not able to make

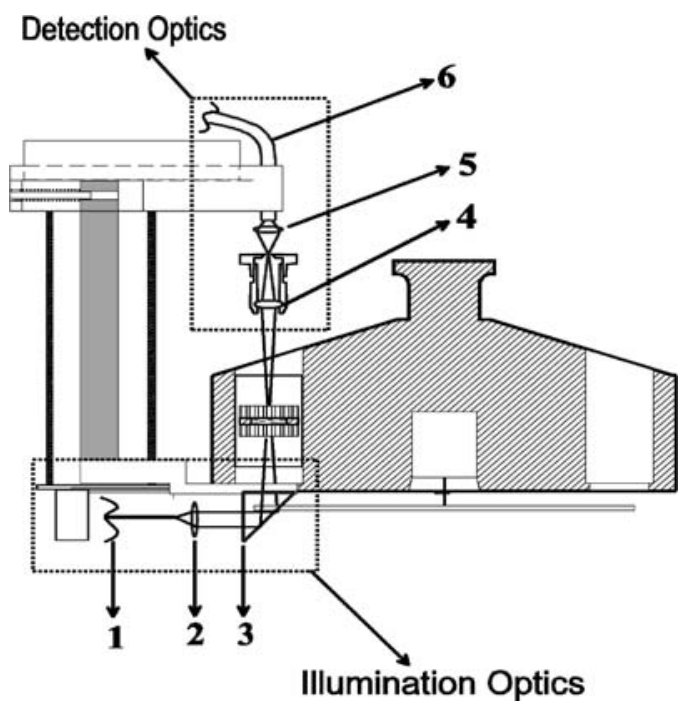

Fig. 3 The detector arm. (1) 600 micron patch fiber UV/Vis (Ocean optics), (2) The collimating lens system (self built), $f=20.6 \mathrm{~mm}$ biconvex, (3) $90^{\circ}$ Quartz prism, (4) Slit-lens assembly (from the $\mathrm{XL}-\mathrm{A}$ ) only one lens $\mathrm{f}=20 \mathrm{~mm}$ biconvex, (5) Focuser, one biconvex lens $(15 \mathrm{~mm})$, (6) $600 \mu \mathrm{m}$ patch fiber (Ocean optics). The light path is also shown schematically

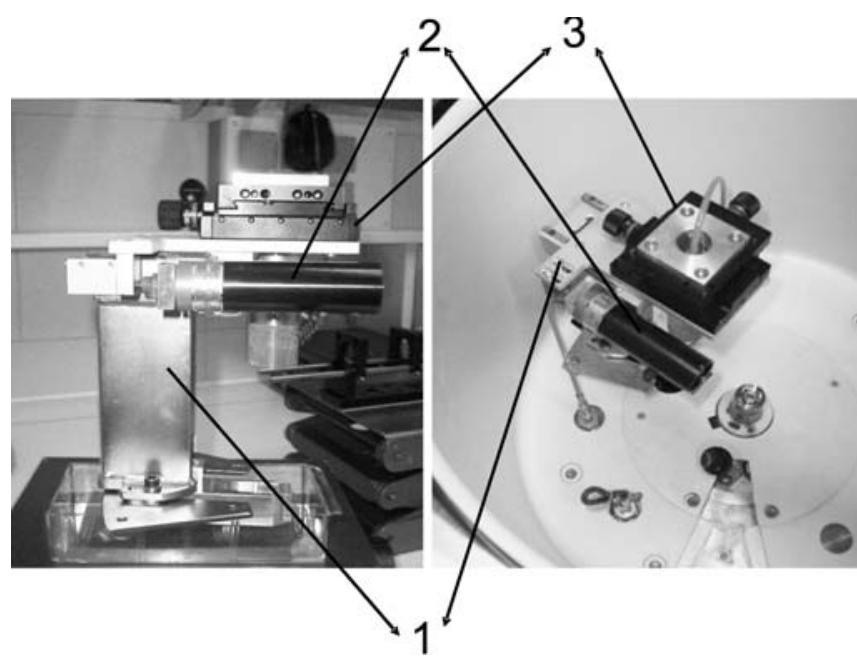

Fig. 4 left. Photograph of the arm, (1) Detector arm, (2) Stepping motor $(0.1 \mu \mathrm{m}$ resolution at up to $4 \mathrm{~mm} / \mathrm{s})$, (3) Detection unit with $x-y$ positioning screws. Right: Mounted arm in the rotor chamber

the light parallel, which is the desired situation and in addition needed as much light for detection as possible to investigate the principal capabilities of this first generation prototype. Earlier efforts were made for producing collimated light by using plano convex lenses in the collimation optics part. However, the desired collimated light could not be achieved by using commercially available lenses. The light, which has passed the centrifuge cell is then collected by a modified slit-lens assembly from a Beckman XL-A (4 in Fig. 3) with a $25 \mu \mathrm{m}$ slit forming the aperture for the radial resolution. The camera lenses of the original system were replaced by a single biconvex lens $(\mathrm{f}=20 \mathrm{~mm})$. After the light has passed the slit, it is collected by a commercial focuser system (Ocean optics, with a $\mathrm{f}=15 \mathrm{~mm}$ biconvex lens) and coupled back into a fiber to the spectrometer. The whole slit-lens assembly can be adjusted by an $x-y$ table to collect the maximum light.

The entire illumination and detection parts of the detector arm (Fig. 3) are connected so that they move as a unit along the ultracentrifuge cell. This is achieved by a Zaber (Zaber, Richmond, Canada) step motor (Model T-LA28-SV), which is very fast and precise (Fig. 4). It enables a scanning speed of up to $4 \mathrm{~mm} / \mathrm{s}$ with an accuracy of $100 \mathrm{~nm}$. In principle, the motor can scan a whole cell in only $3 \mathrm{~s}$. The motor axis itself pushes a plate attached to a spring to ensure mechanical contact between the step motor and the moveable part of the detector arm. The whole detector arm is shown in Fig. 4 in the unmounted state and mounted in the ultracentrifuge.

The UV-Vis Spectrometer. The most important part of the UV-Vis optics, besides the detector arm, is the detector itself. In the present study, we have applied a USB2000 spectrometer (Ocean Optics, Duiven, The Netherlands). There are many available spectrometer options (see http:// 
oceanoptics.com/), the most important being the choice of the diffraction grating and the spectrometer aperture (see Fig. 5). We have applied the 1200 line holographic grating for the UV optimized spectrometer \& 600 lines blazed grating at $400 \mathrm{~nm}$ for the UV/Vis in combination with a $25 \mu \mathrm{m}$ slit (for UV/Vis) and a $50 \mu \mathrm{m}$ (for UV) entrance slit as the entrance aperture (Fig. 5, component 3). The main feature of the USB2000 UV-Vis spectrometer is the use of a CCD line array detector (a 2048-element linear silicon CCD array), which allows for the simultaneous detection of the entire spectral range within an integration time as short as $3 \mathrm{~ms}$. Practical integration times used in this study are in the $50-100 \mathrm{~ms}$ range so that the spectrometer is rate limiting rather than the lamp.

The USBS2000 spectrometer has several vital components. They are shown in Fig. 5 and explained in the following. First, the fiber with the light coming from the sample cell is connected to the SMA905 connector (1) of the spectrometer. After passing a filter (2), the light passes the 25 or $50 \mu \mathrm{m}$ slit (3) as the entrance aperture. It is then collimated by a collimating mirror (4), which reflects the light from the entrance aperture as a collimated beam towards the diffraction grating (5), which disperses the white light. The refracted light is subsequently reflected by a focusing mirror (6), which reflects and focuses the light onto the detector array. The light collection efficiency is further increased by a cylindrical lens in front of the CCD array (7). Before the light finally reaches the CCD detector (9), it passes through a longpass filter (8) for the elimination of second and third order effects. All spectrometer lenses were coated with a special coating for increased UV transmittance. The spectrometer data can then be directly read out by the commercial spectrometer software. However, this is only of very limited use for the application in an AUC. We therefore used the LABVIEW drivers supplied by Ocean Optics and programmed our own application software (see 2. Software developments).

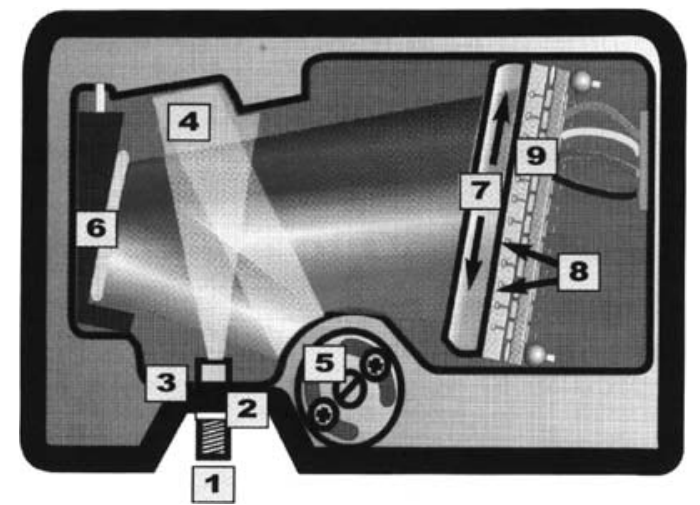

Fig. 5 The USB2000 UV-Vis spectrometer. Figure courtesy of Ocean Optics and reproduced with kind permission from Ocean optics BV, Duiven, The Netherlands. For description of the components see text
Multiplexer and Data Acquisition System. To control the firing of the lamp, a multiplexer system is needed. For this, a trigger signal per rotor revolution has to be generated. This is achieved in our case by polishing an inner sector shaped part of the rotor next to the overspeed disk. This polished part is detected by a reflection light gate similar to an earlier system developed for the model E AUC [42,44] resulting in a TTL rectangular trigger pulse per rotor revolution. This TTL trigger signal then serves for the precise determination of the rotor speed and the exact time between two rotor revolutions. This time interval is divided into 1000 steps corresponding to an angular resolution of $0.36^{\circ}$ on the spinning AUC rotor and the time delay of a cell passing the optical detection part to the trigger signal from the rotor has to be calculated by a so-called multiplexer. These units were described before as analog and partially already as digital devices to enable the use of multiplace rotors without wedge windows; [14-17,45-55]. Multiplexer technology is also common standard in the XL-I AUC although for this centrifuge platform, it is difficult to get precise information on the position where to grab a TTL output signal from the multiplexer. As such signals have to be grabbed directly from the XL-I boards, it is recommended that the user supplies their own separate system for the generation of a TTL trigger pulse similar to the one used in this study.

As we aim at a complete software control of the centrifuge system, we applied a National Instruments PCI6602 timer card, which can be programmed under the LABVIEW environment using the supplied virtual instrument drivers. The programming of the multiplexer is described under Software developments.

\section{Principal Operation Modes}

The new fiber based UV-Vis detection system was developed for two principal operation modes. One is the familiar radial mode, where the radial concentration variation in the sample cell is observed at various times, as already realized in all previous AUC's (Radial mode). This mode, however is not suitable for very polydisperse systems, and only very recently, it became possible to combine the radial profiles obtained at various speeds for a sedimentation velocity experiment $[56,57]$.

For very polydisperse samples, the use of speed profiles is essential to resolve the complete sample over the entire colloidal range $[7,58]$. This is achieved by observing the sample at a fixed position over time so that via use of a speed profile, all samples pass the detector at some stage and are detected (Time mode). This mode is not only interesting for colloids but also for polymers, for example for the detection of aggregates etc. In addition, it has to be stated that the time mode is a very universal mode, where several different samples can be combined in one run, simultaneously enhancing the effectivity of the AUC measurements. 
For each of the detection modes, a calibration scan has to be performed to find the correct sector position as well as for the calibration of the radius. The cell position calibration is done fully automatically, as is the radial calibration. An option is implemented to store the calibration file and use it if the same rotor and cells are used again, which is especially useful for fast sedimenting samples. Alternatively, an option can be selected to perform the radial calibration after the last scan of the experiment, which allows for instantaneous sample observation but with the disadvantage that the detector positions are not precisely known until the calibration step at the end of the experiment. Both modes are restricted in speed by the flash repetition speed of the flash lamp.

Time Mode. In this mode, the detector is set fixed at a defined and known position in the cell. Usually, this is the center of the cell, but if a short experimental duration is desired, a position near the top of the cell can be selected, where care must be taken that the detection position is far enough from the meniscus to avoid optical and physical distortions of the sedimenting boundary. If the highest fractionation of the sample is desired, a position near the cell bottom can be used. As the detector is very fast, it is possible to use more than one detection positions, as the motor of the detection system can move $4 \mathrm{~mm} / \mathrm{s}$ and the acquisition of a spectrum is in the ms range. With this, the movement of the sample can be followed between two or more precisely known positions, which greatly enhances the accuracy of measurements in the time mode, as already discussed for the dual beam mode [59]. Up to now, in the existing turbidity optics $[8,9]$, the sample is detected at a single position and the cells have to be completely filled in order to know the starting point of sedimentation, with the related inaccuracies in case of small remaining air bubbles etc.

In the time mode, the scan time interval is set as well as the radial position for detection and the length of the entire experiment. This mode is not only useful for very polydisperse samples but also for very fast detection, as a sedimentation velocity experiment can be performed in just a few minutes by speeding up the centrifuge to $60000 \mathrm{rpm}$ with maximum acceleration provided that the samples sediment sufficiently fast. This suppresses diffusion to the maximum possible extent. Also, the time mode runs can be very useful screening experiments to find out proper centrifugation conditions for radial scans of an unknown sample. In addition it can be used as supplementary data to radial scan data in a global analysis approach [32], as the time mode can detect minor amounts of large aggregates, which maybe lost from detection during rotor acceleration.

Radial Mode. The radial mode is familiar to every centrifuge user. The described multiwavelength detector essentially works as the XL-A AUC with the difference of a higher speed and a whole acquired UV-Vis spectrum for each radial point. However, if the user is just interested in 1-3 wavelengths for radial scans, for example to be compatible with existing XL-A evaluation programs, they can be selected in analogy to the XL-A with the difference, that for the CCD based detector, it makes no difference in detection speed how many wavelengths are detected. For all other applications, the wavelength range is selected in order to save only useful data and to reduce data storage space required. Radial scan intervals down to $100 \mathrm{~nm}$ can provide a factor 100 in the amount of radial points as compared to the XL-A, which could be a very useful feature for sedimentation equilibrium analysis. Likewise the fast scanning times allow many more radial scan to be acquired per experiment than the XL-A, even with the additional multiwavelength information. However, making the radial steps too small can over-sample the data because two neighbor radial points could be not independent from each other anymore depending on the radial optical resolution of the system.

\section{Software Development}

The software for centrifuge control, multiplexer and data acquisition was written using the LABVIEW 7.1 (National Instruments) programming environment. The software has the capability of ultracentrifuge and step motor control via a serial port, spectrometer and flash lamp triggering via the timer card, as well as data acquisition from the spectrometer. It is convenient to classify software options into three categories such as General Options, Cell Options and Calibration Settings.

1) General Options. Before starting an experiment, some important parameters of the experiment are adjusted.

Rotor Adjustment: 4 hole or 8 hole rotor

XL Settings Adjustment: Speed, temperature and time setting of ultracentrifuge

Scan Mode

Time Mode Scan

Radial Mode Scan

Scan Options Adjustment

Acquire intensity data instead of absorbance: Default is absorbance data

Filter scan intensities below a certain level: Only in radial mode scan

Stop XL after last scan

Make radial calibration after experiment

Method Scan Options Adjustment

Delayed Start: Time delay before the first scan

Time between Scans: In Radial Mode only

Number of Scans: In Radial Mode only

Experiment Directory 
2) Cell Options. For both time mode and radial mode scan, after enabling desired cells, their experimental parameters should be adjusted, which differ in the two modes

Common Options

Wavelength Selection: Up to 4 wavelengths

Saving Full Spectra

Wavelength Range

Time Mode Options

Radius: Position in the cell

Acquisition/Averaging time for one data point (number of lamp flashes)

Radial Mode Options

Rmin and Rmax: Minimum and maximum radial distance

Radial Step: Radial step size between two detector readings

Replicates: Number of data points collected at each wavelength

Set All Settings Identical to Cell 1: Sets the parameters of cell 1 for each cell

3) Calibration Settings.

Cell Calibration: used to set the angular cell positions with respect to the triggering pulse.

Position: angle from the trigger pulse to the cell

Length: size of the cell in angle units

Shifting:provides to access two different positions in the cell e.g. reference and sample sector to allow for the correction of the sample for the reference solvent

Angle Calibration: the positions of the cells can be determined with this property

Radial Calibration: inner and outer radii for the cells are determined

Step Motor Settings: Adjustment of Port, Minimum and Maximum positions of motor

Calibrate Outer Position: Maximum outer position of the step motor determined with a sensor on arm

Graph Settings: Determination of Scale Settings

When the scan starts, the user can observe the experiment online for each cell. Some important information is also shown such as XL Status and Cell Information. It is also possible to change some experimental parameters during the scan such as XL speed, temperature and the intensity limit below which filtering is done. This is an important improvement with respect to the commercial XL-I, which only allows for parameter changes after stopping an ongoing scan. All the options above can be saved in different files for different categories so that the user only needs to adjust necessary parts for any other experiment.

The user interface of the control system was adapted to the style of the existing XL-I centrifuge control user interface to maintain an easy operation by the majority of users, already being familiar with the XL-I instrument control and data acquisition software.

\section{Basic System Performance}

The basic check if the optical system correctly images the ultracentrifuge cell and also provides sufficient radial resolution can be performed by scanning a slit. We used a $200 \mu \mathrm{m}$ slit, which was placed as coating on a cell window (see Fig. 6 lower image). This window was used in an AUC cell with a $2 \mathrm{~mm}$ centerpiece with the slit facing the centerpiece and oriented perpendicular to the radial direction. Scanning this cell in the intensity mode yields a radial scan, which should show a $200 \mu \mathrm{m}$ wide rectangular peak. Ideally, the transition from zero to maximum intensity should be very sharp. However, as the aperture slit for the optics is only $25 \mu \mathrm{m}$, a better optical resolution cannot be expected. In Fig. 6, the result of this experiment is shown for the XL-A (right) and our fiber detector (Fig. 6 left). For the experiment, we chose a radial step size of $3 \mu \mathrm{m}$, the step size for the XL-A was set to the finest possible value of $10 \mu \mathrm{m}$. It can be seen that the transition zone for $0-100 \%$ intensity is about $50 \mu \mathrm{m}$ for the fiber optics detector. Due to the limited number of data points, the transition is more shallow for the XL-A optical system. This demonstrates that our basic optical system performance is at least as good as that of the XL-A, although we use a lens optics, which shows the chromatic aberration problem for white light.

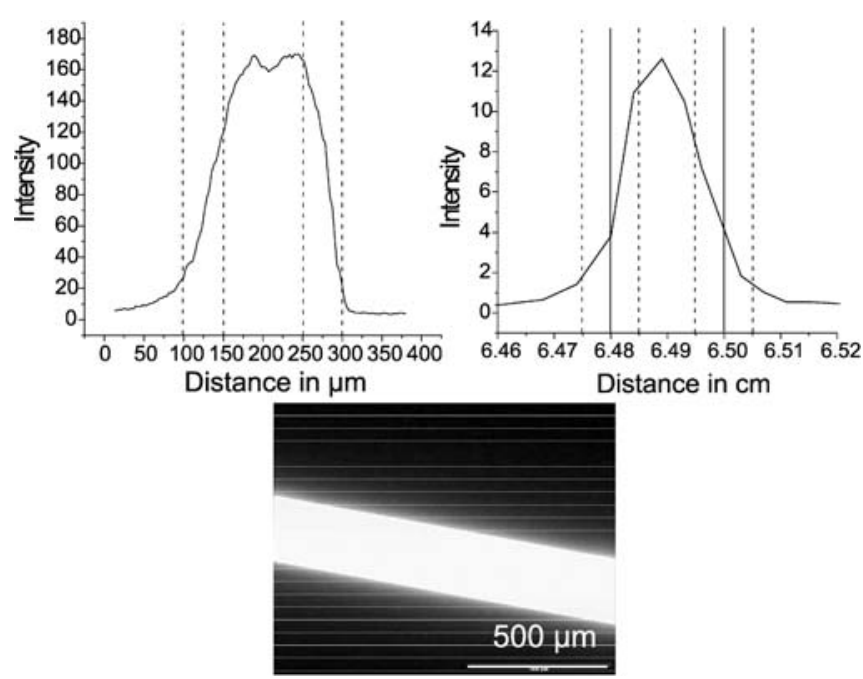

Fig. 6 Resolution observed by placing a 200 micrometer slit at the centre of the cell. The figure in the top left shows observation for the fiber optics based detector. The figure at upper right shows the observation for the commercial XL-A. Bottom: A light microscopy image of the slit. Scale bar $=500 \mu \mathrm{m}$ 


\section{Results and Discussion}

Time Mode. The first sample, which we used to test our optical system was bovine serum albumin (BSA) - a standard protein sample which shows an absorbance peak at $280 \mathrm{~nm}$ in the UV-Vis spectrum. We set the slit position to $6.1 \mathrm{~cm}$ close to the meniscus in order to enable a fast measurement in the time mode. Absorbance was determined by recording the intensity of the sample and applying the intensity recorded for the reference solvent in a separate calibration experiment. The experimental raw data are shown in Fig. 7. The tremendous amount of data becomes directly obvious. Also, it can be seen that the present lower wavelength limit of the fiber detector is $240 \mathrm{~nm}$, which just shows the onset of the $230 \mathrm{~nm}$ BSA peak. This is a bad situation for investigations of proteins, where usually the $230 \mathrm{~nm}$ peak is the most intense one but tolerable for most other samples. If only one wavelength is picked out of the multiwavelength scans, the time dependent sedimentation can be visualized in a similar way to the traditional AUC scans and the data quality can be controlled (Fig. 8). The expected sigmoidal curve is shown and the data quality is comparable to that of the XL-A.

Repetition of the experiment in time mode with the fiber optics and in the radial mode in the XL-A yielded comparable results in terms of the sedimentation coefficients for the identical sample run on both instruments as shown in Fig. 9.

The noise level in both scans is about the same although for the fiber optics, there are many more data points, which is advantageous for the subsequent evaluation. Another obvious difference is the broadness of the experimental curve. The apparent sedimentation coefficient distribution, which is not corrected for diffusion broadening is considerably sharper from the fiber optics than from

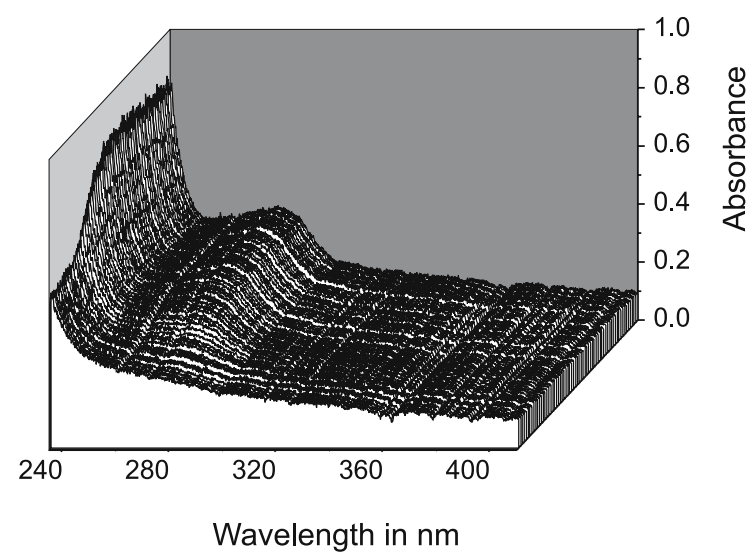

Fig. 7 Sedimentation velocity experiment for BSA, temperature: $25^{\circ} \mathrm{C}$, Rotor Speed: $50000 \mathrm{rpm}$, Slit position: $6.1 \mathrm{~cm}$, lowest detectable wavelength $240 \mathrm{~nm}$. Spectra recording condition: $98 \mathrm{~ms}$ integration time and 10 averages

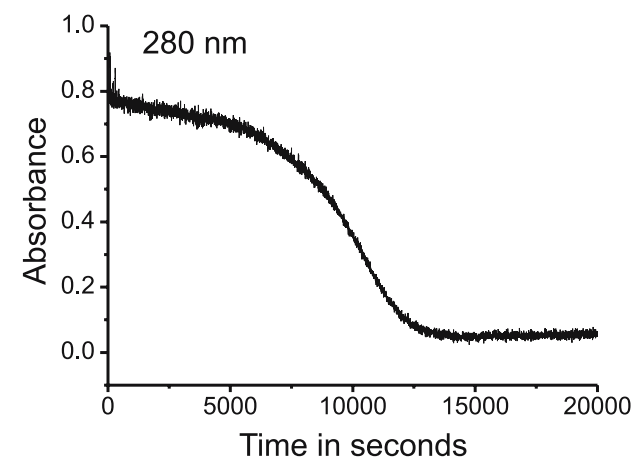

Fig. 8 Absorbance vs time plot for BSA $1 \mathrm{~g} / \mathrm{L}$ at $280 \mathrm{~nm}$. (Experimental conditions; Rotor speed: $5000 \mathrm{rpm}$, Slit position: $6.1 \mathrm{~cm}$. Data recorded with $98 \mathrm{~ms}$ integration time and 10 averages). The data were not yet corrected for reference solvent

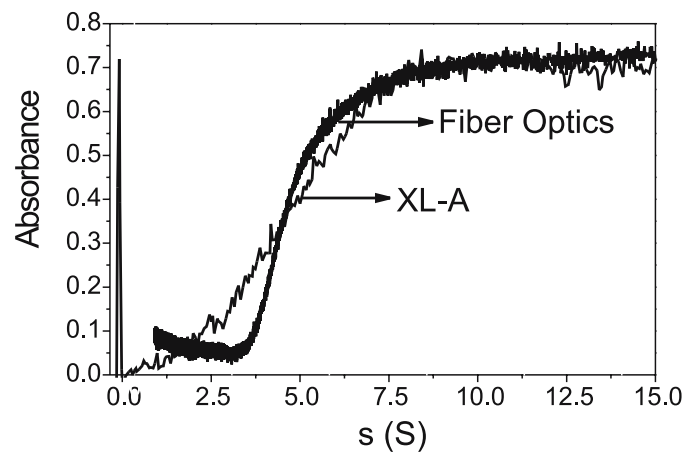

Fig. 9 Comparison of XL-A and Fiber optics. Slit position $6.1 \mathrm{~cm}$, Meniscus $5.81 \mathrm{~cm}$, Fiber optics: $1 \mathrm{~s}$ time interval and time mode scan, XL-A: Continuous radial scan $0.05 \mathrm{~cm}$, 2averages. For fiber optics: 14100 points and for XL-A: 250 points. The data were recorded at wavelength $280 \mathrm{~nm}$. Equation used to convert ' $r$ ' values to ' $\mathrm{s}$ ': $\mathrm{s}=\left(1 / \omega^{2} \mathrm{t}\right) \ln \left(\mathrm{r} / \mathrm{r}_{\mathrm{m}}\right)$; variables have their usual meaning

the XL-A. This effect is attributed to the fact that the detector was placed very close to the meniscus so that diffusion broadening of the boundary could not yet set in. Whereas the sedimentation coefficient distributions get sharper with time in the radial mode, as diffusion goes with the square root of time but sedimentation is directly proportional; this is not observed in the time mode as long as the detector is placed at a position, where diffusion broadening could not yet set in to a significant extent. On the other hand, the fractionation capability of the AUC is not fully exploited with that setting. A slightly tilted lower plateau is observed for the data from the fiber optics. The reason for this is not yet clear, but it has to be stressed that we do not yet use a reference solvent correction nor a correction for variations in the flash lamp intensity. In addition, this tilting of the lower plateau is not regularly observed.

Another comparison between the time mode of the fiber optics detector and the radial mode in the XL-A was made for a mixture of three latex standards $(150 \mathrm{~nm}$, $190 \mathrm{~nm} \& 240 \mathrm{~nm})$. The results are shown in Fig. 10. 

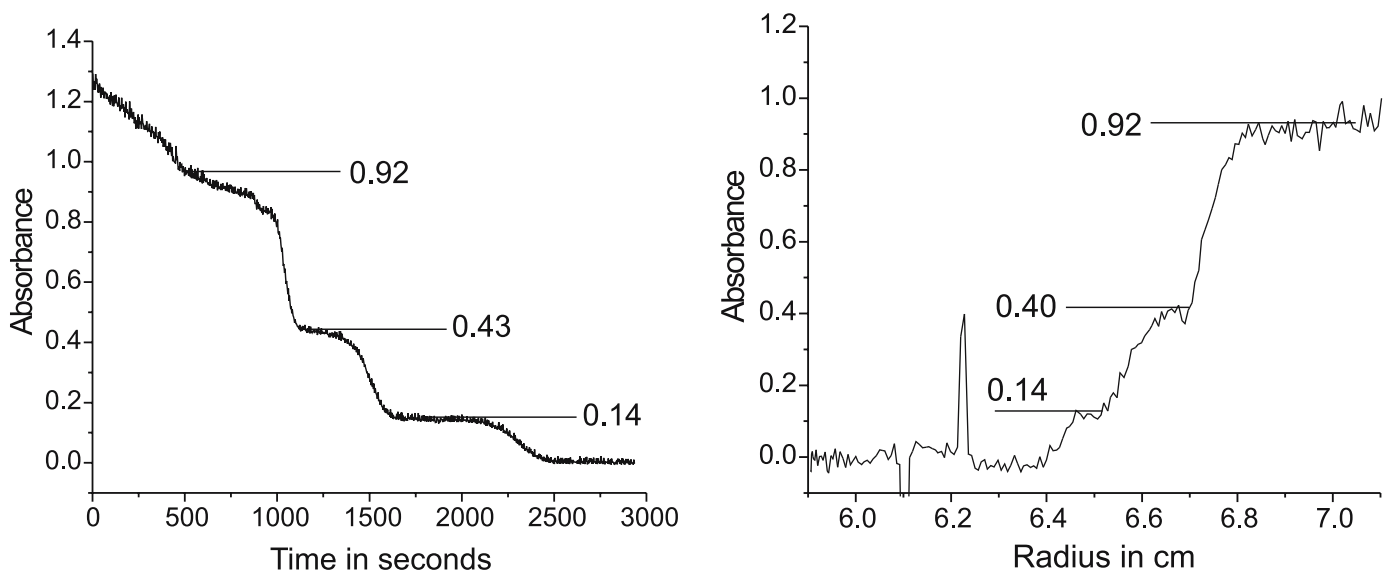

Fig. 10 Sedimentation velocity of a latex mixture (Polystyrene latex mixture with particle sizes $150 \mathrm{~nm}, 190 \mathrm{~nm}$ and $240 \mathrm{~nm}$ ). Left: Fiber optics, time mode scan (slit position $6.1 \mathrm{~cm}$, Recording conditions, wavelength: $450 \mathrm{~nm}$, Integration time: $100 \mathrm{~ms}, 10 \mathrm{averages).} \mathrm{Right:}$ $\mathrm{XL}-\mathrm{A}$, radial mode scan, continuous $(0.005 \mathrm{~cm}$ and 2 averages)

It can be seen that the 3 components are resolved in both ultracentrifuges. Also, the detected concentration of the individual species is comparable for both ultracentrifuges. The data quality of the fiber optics detector is, however, much higher than that of the XL-A. The reason maybe the fact that the flash lamp intensity is lower in the visible range, which degrades the data quality. For the fiber optics, the UV-intensity is significantly decreased due to fiber bending (see discussion below) so that the data quality is better in the visible range for the present prototype. However, for the fiber optics, a significant tilting of the upper plateau is observed at the early times. Such tilting is always observed in case of turbid samples and could have to do with the significantly enhanced stray light for these samples. In the slit-lens assembly of the XL-A, an aperture is installed, which limits stray light from the sample to $+/-4^{\circ}$. This aperture is not yet realized for the present fiber optics prototype. Indicative for stray light is also the fact that just the upper plateau is tilted but not the lower plateau, where all turbid latices have already sedimented.

The turbidity of colloidal dispersions is often a problem for their analysis by AUC. This is caused by the fact that the scattering of particles is size dependent according to the MIE theory where a small amount of large particles scatters more light than a large amount of small particles. This in turn affects the apparent light absorption and turbidity and makes the absorption optics sensitive to size dependence analysis. Therefore, a so called coupling technique was developed for the determination of particle size distributions with a turbidity detector, where the same sample is investigated at a high concentration to detect the small particles and at a low concentration for the detection of the larger particles [60]. This obstacle is omitted with the new multiwavelength detector, as the short wavelengths are especially sensitive, so that small particles could be detected at short wavelengths and the larger ones at the more insensitive large wavelengths in a single experiment. This effect is demonstrated in Fig. 11 for a binary latex mixture, which is displayed for three different wavelengths.

The drastic effect of the wavelength dependence of the detected signals is clearly visible. When for example the wavelengths $430 \& 660 \mathrm{~nm}$ are combined, the two different latexes are detected with a comparable sensitivity.

Another feature of the new detector is its speed. It is no problem to perform a whole sedimentation velocity experiment during the acceleration of the AUC to $60000 \mathrm{rpm}$ with maximum speed provided that the samples sediment fast enough. This is shown in Fig. 12 where the experimental raw data and the differential distribution being proportional to the particle size and sedimentation coefficient distribution are displayed.

In only 3 minutes, the whole sedimentation coefficient distribution is determined. The huge advantage of such experiments beside the speed is the fact that diffusion broadening is suppressed to the maximum possible extent so

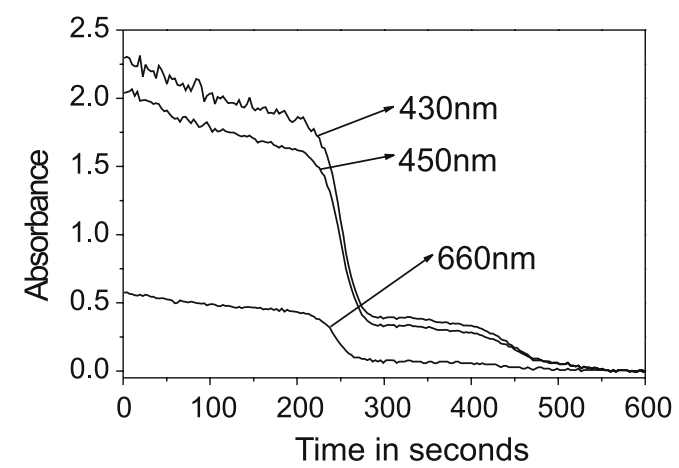

Fig. 11 Latex mixture $(150 \& 240 \mathrm{~nm})$ observed at three different wavelengths $(430,450 \& 660 \mathrm{~nm})$. Experimental conditions: Rotor speed: $10000 \mathrm{rpm}$, Temperature: $25^{\circ} \mathrm{C}$, Slit position: $6.1 \mathrm{~cm}$. Data collection conditions: $100 \mathrm{~ms}$ averaging time, 10 averages 

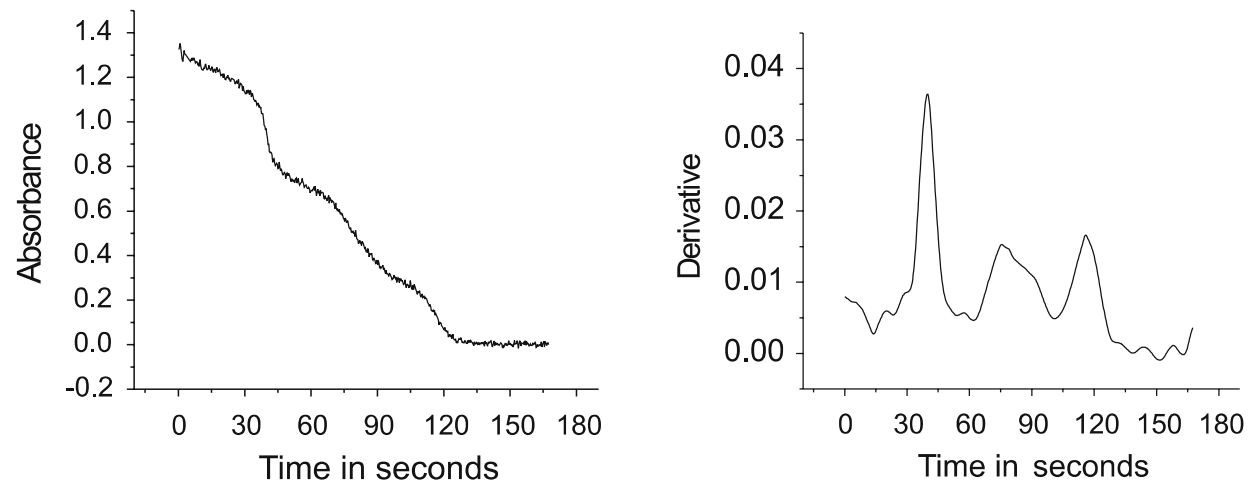

Fig. 12 Fast sedimentation velocity Experiment with a Polystyrene Latex Mixture of sizes $190 \mathrm{~nm}, 305 \mathrm{~nm}$ and $605 \mathrm{~nm}$ in $1: 2: 2 \mathrm{mixing}$ ratio by wt.; data collected at $350 \mathrm{~nm}$; Left: Experimental raw data. Right: Derivative curve was generated performing differentiation after 5 point FFT smoothing. Slit position: $6.1 \mathrm{~cm}$
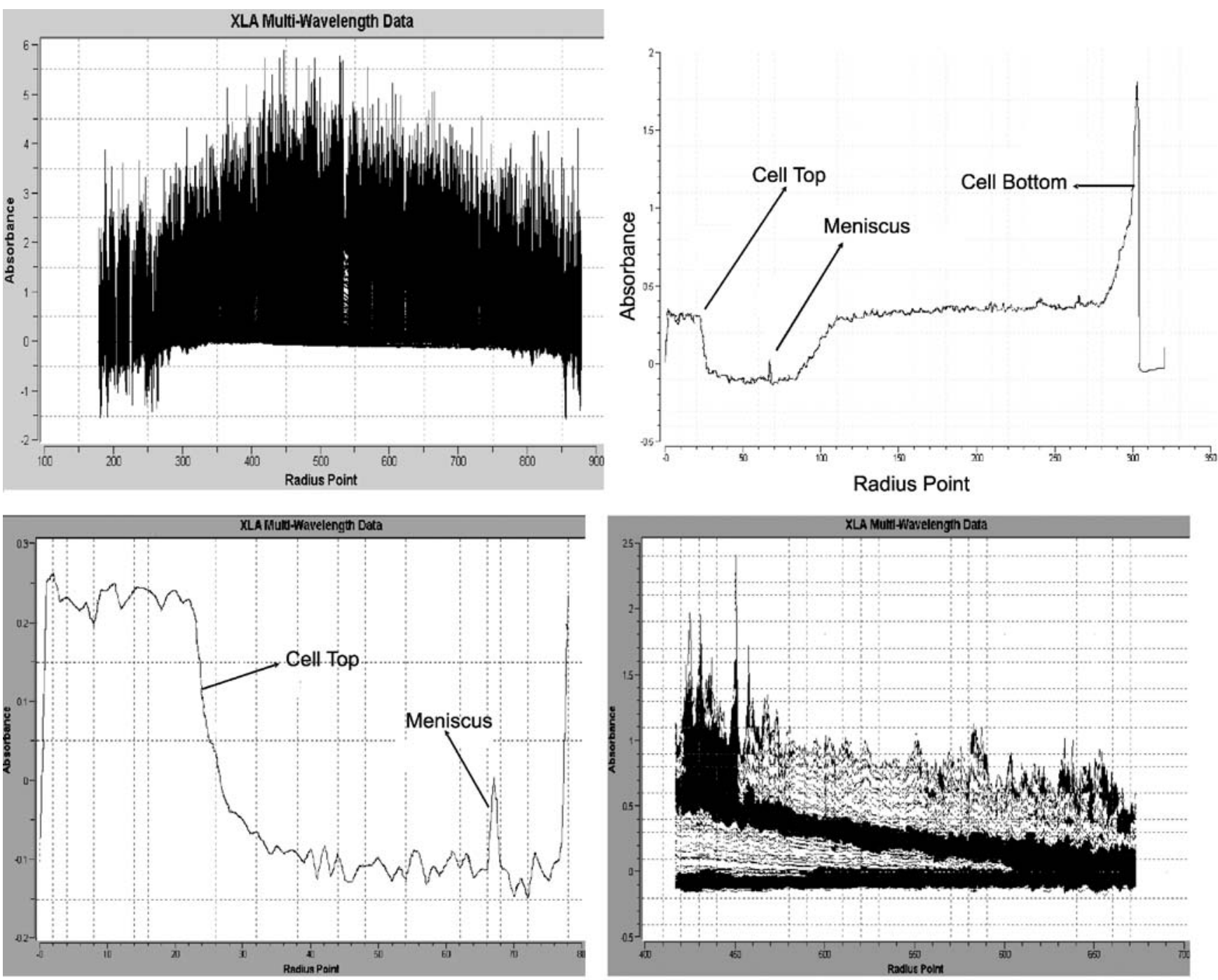

Fig. 13 Upper left: Raw data for a total radial Scan (Sample: $175 \mathrm{~nm}$ Polystyrene Latex, $10000 \mathrm{rpm}, 25^{\circ} \mathrm{C}$ ). Upper right: radial scan at a fixed wavelength. Lower left: Radial scan at a fixed wavelength (Zoomed view), lower right: Radial scan after cutting off noisy regions. Note that the radius is expressed in radius points corresponding to the steps of the detector movement and no real radial calibration was performed yet 
that a diffusion correction can become obsolete for many samples. However, the evaluation of such experiments has to be improved. With this, we can adapt the experiences with the turbidity detector where similar problems were faced.

Radial Mode. The data amount of the radial mode is much higher than that of the time mode, as a whole spectrum can be saved for every radial point. A $1 \mathrm{~cm}$ solution column can in principle be scanned with $100 \mathrm{~nm}$ resolution, which corresponds to $10^{5}$ data points per scan. For radial scans with the detector prototype, we first used the spectrometer software supplied with the spectrometer and visualized the data with a data viewer written by B. Demeler, UTHCSA, San Antonio. In the following we demonstrate how the useable data in a single scan can be extracted for the example of a $175 \mathrm{~nm}$ latex sample (Fig. 13).

First of all, all experimental data are read in for a particular scan. If the whole cell is scanned as in this example, the first view along the cell from top to bottom just shows noise from the cell part before the sector starts (Fig. 13 upper left). Therefore, the plot has to be transformed from the wavelength display to a radial display at a selected wavelength (Fig. 13 upper right), which is a familiar data display for every AUC user.

In the zoomed view (Fig. 13 lower left) the meniscus can be seen. Also top and bottom of the cell are clearly visible and define the data regions, which have to be cut off. This step is eliminated in the new data acquisition software, where the radius is calibrated before the experiment, so that only the useful data range is scanned. Also, noisy wavelength regions can be clipped until the final scan is obtained as shown in Fig. 13 lower right. If this scan is turned appropriately, the three dimensional nature of a scan becomes obvious (Fig. 14).

Not only the sigmoidal radial sedimentation velocity profile is shown, but also the wavelength dependence of

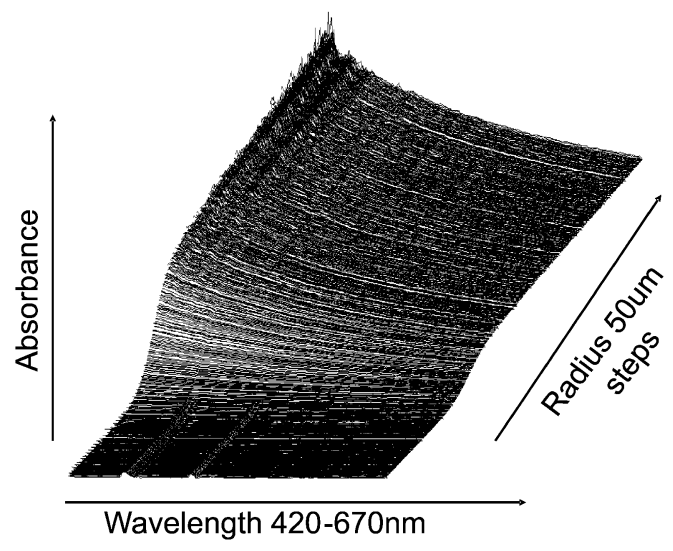

Fig. 14 Radial Scan for a $175 \mathrm{~nm}$ polystyrene latex sample (10000 rpm, $25^{\circ} \mathrm{C}$ )

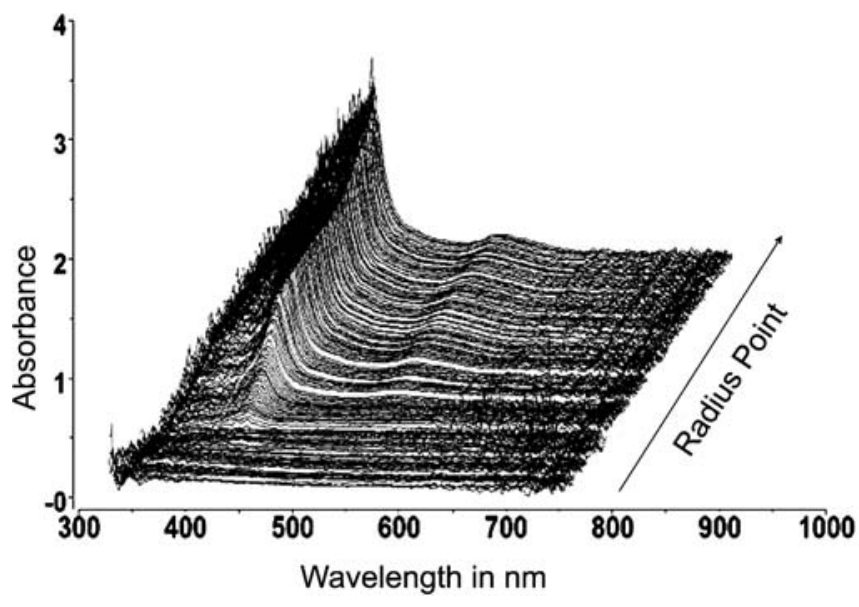

Fig. 15 Radial scan for a $1.0 \mathrm{~g} / \mathrm{L}$ solution of Cytochrome C (Speed $50000 \mathrm{rpm})$

turbidity, which can even be used to evaluate the colloid particle size independently. It is clear that such scan contains much more information than the conventional two dimensional radial scan. Especially fitting algorithms should greatly profit from the additional wavelength dimension. Another example for a single radial scan is presented in Fig. 15 for Cytochrome C. Even although the typical $230 \& 280$ protein absorption peaks could not be detected without significant noise in this example, Fig. 15 already shows the information increase of a single multiwavelength scan as compared to a radial scan at a single wavelength as performed in the commercial XL-I. The use of the introduced multiwavelength detection can easily be imagined for interacting systems with different chromophores.

Problems. One of the big current limitations of the detector is the low light intensity in the UV region, which is the most important part of the spectrum - at least for

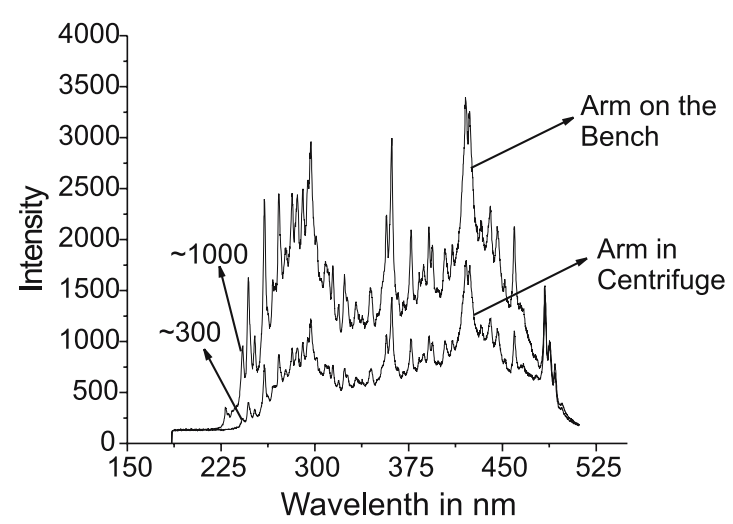

Fig. 16 UV-Vis spectrum detected without fiber bending on an optical bench and with fiber bending in the AUC 
many biopolymers. This is mainly caused by the necessity to bend the fibers upon installation of the detector arm in the ultracentrifuge (see also Fig. 4). Fiber bending reduces the light especially in the UV region $<250 \mathrm{~nm}$. When the detector arm is mounted on an optical bench without fiber bending, there is still an intensity of more than 200 counts registered down to $225 \mathrm{~nm}$, which would enable the investigation of proteins. Fiber bending, however, causes intensity loss of a factor of three around $250 \mathrm{~nm}$ and about a factor of two for the rest of the spectral range (Fig. 15). Therefore, the construction of the arm has to be modified to avoid fiber bending.

\section{Conclusion and Outlook}

In this work, we could show a first prototype of a fiber based multiwavelength detector for the XL preparative ultracentrifuge. First measurements demonstrate that the detector is very fast with present typical scan times of only $100 \mathrm{~ms} /$ spectrum and delivers a data quality comparable to that of the XL-A. Due to the speed of the detector, two experimental modes are possible - the well known radial scanning mode as well as the time mode, where the detector is set at a fixed position and the sedimentation is observed with time. These modes extend the possibility of AUC with absorption optics detection to very polydisperse samples. Sedimentation velocity experiments as fast as two minutes become possible now, where just the centrifuge is accelerated to $60000 \mathrm{rpm}$ and immediately slowed down again. The detector design was made in a modular way so that all components can be easily exchanged. Therefore, advantage of new developments for example in lamp or spectrometer performance can be fully taken. For example, Ocean optics has very recently released the HR4000 spectrometer, which can scan as fast as $10 \mu \mathrm{s}$. This already allows the use of a continuous lamp with a pulsed spectrometer so that a very high light intensity could be realized in future designs on that basis. As commercially available compounds are applied, the price for the detector is also moderate.

One drawback of the present detector is the high light intensity loss upon fiber bending and the use of lens optics with the associated chromatic aberration problem. Although the latter appeared to be of minor importance, we will develop a mirror optical system for this detector in the future, which enables defined and equal light beams at all wavelengths. In addition, mirrors can help to eliminate the necessity for fiber bending.

Nevertheless, the present detector prototype is a cheap and modular add on, which transforms a preparative ultracentrifuge into an analytical machine. The now available multiwavelength data add a further dimension to ultracentrifuge analyses allowing for future use of global multiwavelength fits, evaluation of the particle size via the wavelength dependence of turbidity, a turbidity detection with multiple sensitivity, rapid sedimentation velocity experiments and many more. Nevertheless, the software basis for these applications still needs to be developed.

Acknowledgement We thank the BASF AG, Ludwigshafen and the Max-Planck-Society for financial support of this work. We also thank Dr. Walter Mächtle, BASF, for useful discussions and Dr. Borries Demeler, UTHCSA, San Antonio for writing a data visualization program to display the multiwavelength data. The mechanical workshop of the BASF is acknowledged for the modification of the XL heatsink and for building the basic detector arm. Andreas Kretzschmar, mechanical workshop MPI-KGF, is acknowledged for multiple modifications of the detector arm. Henryk Pitas, electrical workshop MPI-KGF, is thanked for help with electrical problems. Dr. K. Tauer (MPI-KGF) is acknowledged for the latex samples and Dr. Neil Robinson (UTHCSA) for the cytochrome samples. Finally, we thank Antje Völkel for helpful assistance and Prof. Dr. Dr. h.c. Markus Antonietti for the overall support of this project.

\section{References}

1. Svedberg T, Pedersen KO(1940) The Ultracentrifuge. Clarendon Press, Oxford

2. Lloyd PH (1974) Optical methods in Ultracentrifugation, Electrophoresis, and Diffusion: with a guide to the interpretation of records. Clarendon Press, Oxford

3. Schmidt B, Riesner D (1992) A Fluorescence Detection System for the Analytical Ultracentrifuge and its Application to Proteins, Nucleic Acids, Viroids and Viruses. In: Harding SE et al. (ed) Analytical Ultracentrifugation in Biochemistry and Polymer Science. Royal Society of Chemistry, Cambridge, p 176
4. MacGregor IK, Laue TM (1999) Biophys J 76:357

5. MacGregor IK, Anderson AL, Laue TM (2004) Biophys Chem 108:165

6. Cantow HJ (1964) Makromol Chem 70:130

7. Scholtan W, Lange H (1972) Kolloid Z Z Polym 250:782

8. Müller HG (1989) Colloid Polym Sci 267:1113

9. Mächtle W (1992) Analysis of Polymer Dispersions with an Eight-Cell-AUC-Multiplexer: High Resolution Particle Size Distribution and Density Gradient Techniques. In: Harding SE et al. (ed) Analytical
Ultracentrifugation in Biochemistry and Polymer Science. Royal Society of Chemistry, Cambridge, p 147

10. Klodwig U, Mächtle W (1989) Colloid Polym Sci 267:1117

11. Clewelow AC, Errington N, Rowe AJ (1997) Eur Biophys J 25:311

12. Holtus G, Borchard W (1989) Colloid and Polymer Science 267:1133

13. Cölfen H, Borchard W (1994) Progr Colloid Polym Sci 94:90

14. Kisters D, Borchard W (1999) Progr Colloid Polym Sci 113:10

15. Laue TM (1992) On-Line data aquisition and analysis from the Rayleigh interferometer. In: Harding SE et al. (ed) Analytical 
Ultracentrifugation in Biochemistry and Polymer Science. Royal Society of Chemistry, Cambridge, p 63

16. Börger L, Lechner MD, Stadler M (2004) Progr Colloid Polym Sci 127:19

17. Mächtle W (1999) Progr Colloid Polym Sci 113:1

18. Flossdorf J, Schillig H, Schindler KP (1978) Makromol Chem 179:1617

19. Giebeler R (1992) The Optima XL-A: A New Analytical Ultracentrifuge with a Novel Precision Absorption Optical System. In: Harding SE et al. (ed) Analytical Ultracentrifugation in Biochemistry and Polymer Science. Royal Society of Chemistry, Cambridge, p 16

20. Flossdorf J (1980) Makromol Chem 181:715

21. Cölfen H, Borchard W (1994) Ultrasensitive Schlieren Optical System. In: Bonner RF et al. (ed) Progress in Biomedical Optics, Vol 2136. SPIE Bellingham, Washington, p 307

22. Schuck P (2000) Biophys J 78:1606

23. Stafford WF (1992) Anal Biochem 203:295

24. Johnson ML, Correia JJ, Yphantis DA, Halvorson (1981) Biophys J 36:575

25. McRorie DK, Voelker PJ (1993) Self-Associating Systems in the Analytical Ultracentrifuge. Beckman Instruments California (http://www.beckman.com/Literature/ BioResearch/362784.pdf)

26. Demeler B, van Holde KE (2004) Anal Biochem 335:279 and Ultrascan Website: http://www.ultrascan.uthscsa.edu/

27. Mächtle W (1991) Progr Colloid Polym Sci 86:111
28. Voelker P (1995) Progr Colloid Polym Sci 99:162

29. Cölfen H, Pauck T, Antonietti M (1997) Progr Colloid Polym Sci 107:136

30. Schuck P (2003) Anal Biochem 320:104

31. Cölfen H, Völkel A (2003) Eur Biophys J 32:432

32. Global analysis is also possible with the SEDPHAT software by Peter Schuck. See the following Website for further details: http://www.analyticalultracentrifugation.com/sedphat/sedphat.htm

33. Vistica J, Dam J, Balbo A, Yikilmaz E, Mariuzza RA, Rouault TA, Schuck P (2004) Anal Biochem 326:234

34. Balbo A, Minor KH, Velikovsky CA, Mariuzza RA, Peterson CB, Schuck P (2005) Proc Natl Acad Sci USA 102 $1: 81$

35. Gledhill RJ (1962) J Phys Chem $66: 458$

36. Bateman JB, Weneck EJ, Eshler DC (1959) J Colloid Sci 14:308

37. Heller W, Bhatnagar HL, Nakagaki M (1962) J Chem Phys 36:1163

38. Hosono M, Sugii S, Kusudo O, Tsuji W (1973) Bull Inst Chem Res, Kyoto Univ 51:104

39. http://oceanoptics.com/technical/ engineering/USB2000\%200EM\% 20Data\%20Sheet.pdf

40. http://oceanoptics.com/technical/ engineering/OEM $\% 20$ Data $\%$ 20Sheet\%20-\%20HR4000.pdf

41. www.lot-oriel.com/ccd

42. Cölfen H (1994) Bestimmung thermodynamischer und elastischer Eigenschaften von Gelen mit Hilfe von Sedimentations-gleichgewichten in einer Analytischen Ultrazentrifuge am Beispiel des Systems

Gelatine/Wasser. Verlag Köster, Berlin

43. Visit the following website: http://usa.hamamatsu.com/en/ products/electron-tube-division/lightsources/xenon-flash-lamps.php

44. Cölfen H, Borchard W (1994) Anal Biochem 219:321

45. Laue TM (1981) PhD Dissertation, Univ Of Connecticut, Storrs, CT, USA

46. Rockholt DL, Royce CR, Richards (1976) Biophys Chem 5:55

47. Laue TM, Domanic RA, Yphantis DA (1983) Anal Biochem 131:220

48. Yphantis DA, Laue TM, Anderson IA (1983) Anal Biochem 143:95

49. Mächtle W, Klodwig U (1979) Makromol Chem 180:2507

50. Mächtle W, Klodwig U (1976) Makromol Chem 177:1607

51. Sedlack U, Lechner MD (1995) Progr Colloid Polym Sci 99:136

52. Ortlepp B, Panke D (1991) Progr Colloid Polym Sci 86:57

53. Schindler KP (1980) Avail NTIS. Report (Order No. PB81-167140): 157

54. Flossdorf J, Schillig H, Schindler KP (1980) J Phys E: Scientific Instruments 13:647

55. Kuhnert R, Boedel E, Stegemann H, Wastl G (1973) CZ-Chem Tech 2:441

56. Müller HG (2004) Progr Colloid Polym Sci 127:9

57. Stafford WE, Braswell EH (2004) Biophys Chem 108:273

58. Müller HG (1989) Colloid Polym Sci 267:1113

59. Mächtle W (1999) Biophys J 76:1080

60. Mächtle W (1988) Angew Makromol Chem 162:35 